\title{
BUILDING SUSTAINABLE MATERIAL NARRATIVES WITH MATERIAL PATHWAYS
}

\author{
Karen Marie HASLING and UIla RAEBLD \\ Design School Kolding, Denmark
}

\begin{abstract}
This paper introduces Material Pathways, a learning tool developed to strengthen awareness, initiate reflection and support discussion on the multifarious roles, materials can have within the design for sustainability field in a common frame. Its objective has thus been to create a common pedagogical ground for notions and concepts used on materials and sustainability in fields such as art and craft, biology, engineering, geography, economy, culture studies and sociology.

The Material Pathways tool is a physical deck of 22 cards, each describing a 'material position' that can be used to unfold understandings of materials in a design project. The deck has been developed as a follow up and complement to the Sustainable Design Cards, a learning tool developed to articulate and activate approaches to sustainable design [1]-[3]. It builds on the same kinds of information and visual layout, making the two decks compatible with each other in the process. Consequently, the decks can be used independently, but also together, to both cover sustainable design broadly and the role of materials herein more specifically.

First, the paper presents the structure of content of each card and second it describes the development of the cards and as a collection. Finally, the paper discusses, how the cards might be used to create a language on and around materials, to frame and guide a process focusing on materials and to strengthen communication of material-driven ideas and intentions.
\end{abstract}

Keywords: Learning tool, material pedagogics, sustainability and design, design education, materials in design

\section{INTRODUCTION}

This paper centres on the Material Pathways deck, a learning tool developed to strengthen awareness, initiate reflection, and support discussion of material roles - or positions as we call them here - in design for sustainability. The motivation for introducing this new tool was to combine, but also to unfold the interdisciplinary fields of "materials" and "sustainability" from a design education and practice perspective.

As developments in the field of design for sustainability grow expansively, materials, as omnipresent as they are, play a vital role and hold the potential to unlock new ways of seeing and being in the world. In contrast to predominantly highlighting technical and environmental aspects, the cards also focus on materials' cultural and emotional values to encourage a critical and mindful approach to working with materials in all stages, from sourcing, to production, using and degradation.

\subsection{Background}

In 2017, we published the Sustainable Design Cards [1]-[3] containing 28 approaches to sustainability in design. Since then, we have experienced how the cards have spread through our network of educators, students, and practitioners and via the website. From the amount of positive feedback, we have received, we can see that the card tool format suits actors in and around design well.

In the Sustainable Design Cards, the perspective on materials is generic and broad to not overpopulate the cards with material related issues. This can be exemplified with having only one card to cover the deep and multidirectional field of 'Environmentally Friendly Materials'. Nevertheless, we knew that there was a need to unfold this area more in detail and beyond 'conventional' approaches such as sourcing organic cotton or using recycled and recyclable plastic. We wished to point towards considerations for materials that relate to a wider range of aspects relevant for design such as the context and use and thereby aspects related to experience and culture, but also aspects that tie these to notions 
such as properties, systems, and time. That is, considering materials in diverse ways when we, as designers, seek to make qualified choices in the shadow of global climate change, social inequality, pollution and decrease of bio-diversity.

The paper outlines the content and structure of each cards and how they have been developed as a collection. As the cards were finalised and published in late 2020, in addition to introducing them in the courses we are engaged with ourselves, we are in the process of distributing them educators, students and practitioners to whom they could be relevant and useful. When doing so, we ask for and hope to receive valuable feedback on, how the cards can be used in different contexts and by different users.

\section{FROM SUSTAINABLE DESIGN CARDS TO MATERIAL PATHWAYS}

From the positive feedback we had received on using Sustainable Design Cards [2], we chose to work with similar structure of content, layout and visual identity for the new deck except for slightly adjusting the intensity of colours used and changing the font. We also saw this as important for emphasising the strong link between the old and the new deck so that it will be both possible and easy to combine and use the two decks interchangeably in a design process.

The card template we have worked from consists of an information side and a graphics side. The information side consists of information on 'What' the material position is about, 'Why' it is relevant to consider in a material in design for sustainability context, possible 'Challenge' with introducing the material position in a design concept, 'Examples' of companies that work the material position, other cards in the card deck that the 'card links to' and 'Further reading' with three to four references for user to read more about the material position.

The graphics side consists of two illustrative means that considers a material position from respectively a material lifetime and a material lifecycle perspective. The two perspectives: one that proposes to prolong the use of materials and products as long and efficient as possible [4] and one that proposes to enable indefinite material and product circulation [5] are often regarded as being in contrast to each other. Nevertheless, we see that the two can co-exist and co-relate, when breaking down the overall complexity.
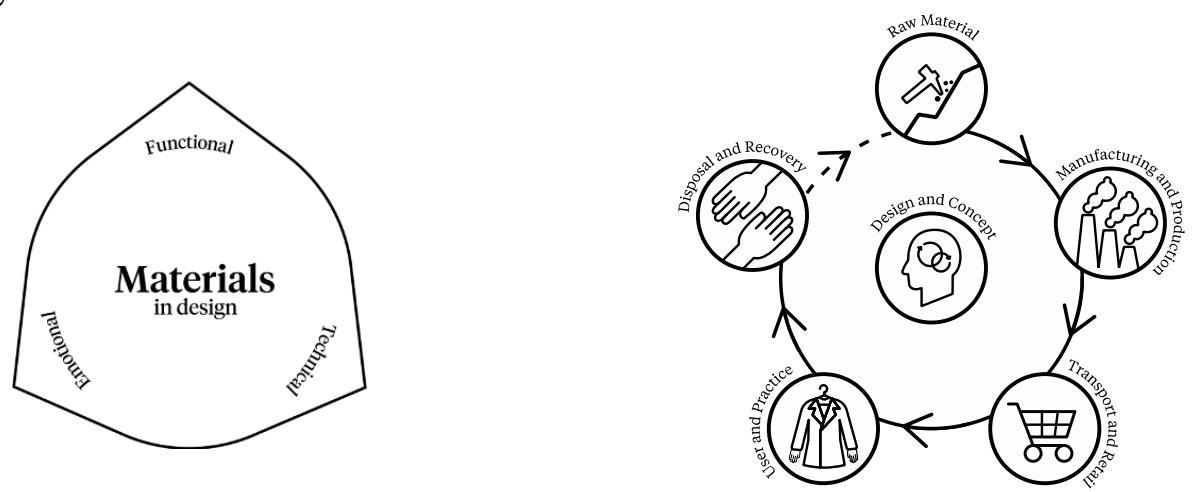

Figure 1. The design compass (left) and product lifecycle (right) applied in the Material Pathways deck

\subsection{The design compass}

Product lifetime can be defined as "the extent of efficient or sufficient use in a product" [4] and can be described by e.g. physical life, functional life, technical life, economical life, legal life and desirability life [6].

In the Material Pathways deck, we specifically consider lifetime from the material perspective and how materials engage in the value creation of the product and facilitate this with a visual compass where the lifetimes are condensed as a tension between technical, functional, and emotional aspects and where. Here we see that:

- Technical aspects refer to those related to the length of time a material or product stays in use before it breaks or wears out

- Functional aspects refer to those related to the length of time a material or product stays in use before its functionality no longer meets the user's expectations or needs

- Emotional aspects refer to those related to the length of time until user loses interest in the material or product 
In the visual model, the compass shown in Figure 4, the material position is indicated as a coloured cloud space rather than a point, as we acknowledge that the understanding of and consideration for a material position will affect its relation to technical, functional, and emotional aspects.

\subsection{The product lifecycle}

In combination with the design compass, six phases illustrated with icon link a material position to a product lifecycle. This feature provides the user with two entrance points to the deck: you may be interested in a particular material position and gain understanding related life cycle phases. But you may also be interested in a specific lifecycle phase, say use phase, and are then able to identify material positions residing in this phase.

The lifecycle phases used in the card decks are:

- Raw Material relates to aspects concerning resource extraction and refining.

- Manufacturing and Production relate to aspects concerning the conversion of resources into products.

- Transport and Retail relate to logistic and handling aspects such as shipping and service concepts.

- User and Practice relate to aspects in use and can be influenced by the user.

- Recovery and Disposal relate to the recovery, reuse, or recycling of a product in post-use.

- Design and Concept relate to aspects in the design and can be integrated by the designer.

\section{WORK-IN-PROGRESS DEVELOPMENT OF CARDS}

Having established a predefined template and frame for cards to be described into and knowing in which direction we were heading, allowed for multiple explorative activities to happen simultaneously.

A first activity was a controlled brainstorming exercise, where we mapped notions that could relate to materials in design for sustainability in the design compass. In that way, we could understand and discuss, which areas that were overrepresented and which ones that needed to be further investigated and developed. This ended up with a preliminary selection of 33 positions that with the help of two student assistants from our intradisciplinary design MA program in sustainability and design were researched and described through individual writing, sharing documents and co-creation sessions for six months.

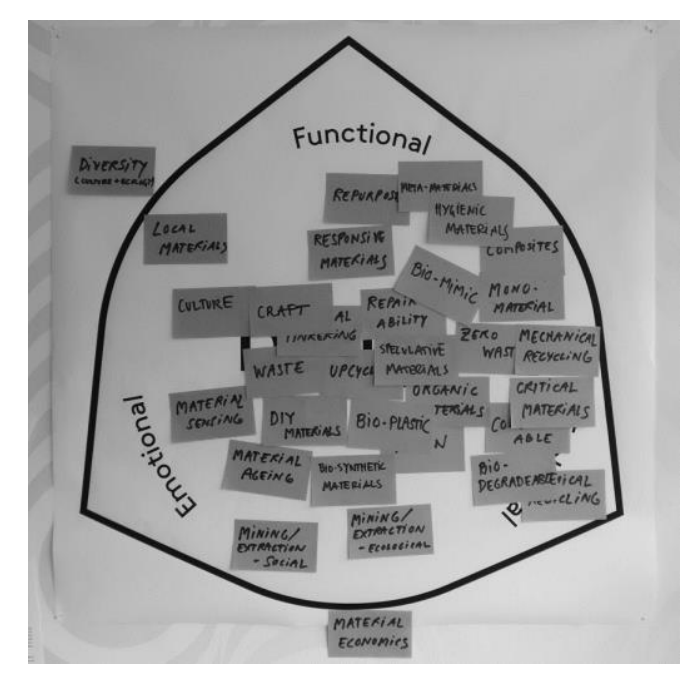

Figure 2. The design compass with preliminary material positions

\subsection{First student engagements with the cards}

The cards are mainly developed for students. In our own institution, the Sustainable Design Cards are introduced on BA-level to make operational sustainability in design. However, with the increasing attention on sustainability in society, the notions and concepts applied are often already familiar to students or some that they can relate to when being expanded on and discussed.

The Material Pathways were believed to possess a higher level of complexity; not necessarily in the logics of material positions themselves, but because they offer and apply a range of new notions and concepts that students have to become familiar with. In that regards, the Material Pathways can be 
regarded as increasing the complexity from the Sustainable Design Cards. Consequently, we saw that students in our MA program on sustainability and design, being used to consider and integrate sustainability in their design projects and knowing and previously having worked with the Sustainable Design Cards, were suitable for 'testing' the first version of the cards.

In November 2019, the Work-in-progress deck of cards was tested in an independent workshop in a course on material narratives with first year MA students. The workshop was conducted as a 2.5-hour session, where students individually and in seven groups of two to four students in each were asked two tasks. To invite students into the process as co-researchers and not only as potential users, but the tasks also aimed to support active discussion rather than solely testing a prototype.

\subsubsection{Task 1: Relatability to proposed material positions}

After a short introduction and being provided with a physical print of the deck students were given the first task. As a way for students to become familiar with the deck and for us to acquire insights on how students would relate material positions to previous work, they were in this first task asked to describe previous projects based on one or more material positions. This was documented in a written template that students were asked to hand in after the workshop (figure 3, left) and was later used to understand, which material positions students were familiar with and had used and which material positions that according to students' work are often combined.
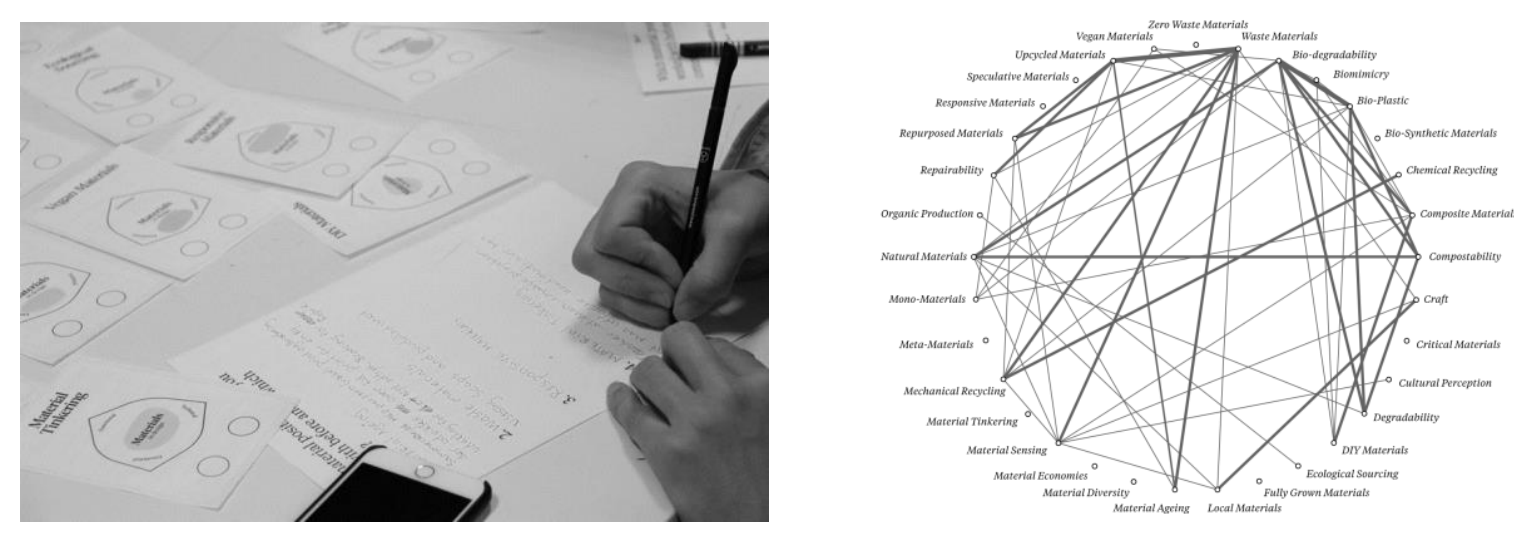

Figure 3. Linking material positions to own previous project work (left) and affinity diagram showing how material positions are linked according to the students (right)

Here the material positions 'Upcycled materials', 'Waste materials, 'Craft' and 'Local Materials', 'BioPlastic' and Mono-Materials' were represented most. It shows a concentration around waste, which makes sense, as waste materials are often accessible to students in that they are very tangible and handson resources that are inexpensive to source and that can be manipulated within the studio or at home. Furthermore, the strong representation of craft and the local can link to the arts and craft root of many design programs including the one we represent.

In contrast, material positions such as 'Bio-Synthetic Materials', 'Fully Grown Materials', 'Material Diversity', 'Meta-Materials' and 'Speculative Materials' were not represented at all. We believe that this can (partly) be because they require particular knowledge or available technology to work with that go beyond what is expected in design education.

Likewise, in an indicative affinity diagram showing students' links between material positions (figure 3 , right), it is evident that the links were not evenly distributed among material positions. This, in addition to insights from the ongoing discussion throughout the workshop, indicated that it would be valuable to reconsider the framing of some of cards, both by means of relevance and relatability.

\subsubsection{Task 2: relating material positions to product lifecycle phases}

In the workshop's second task, in groups students were asked to discuss and assign product lifecycle phases to the card of each material position, this either using the stickers that were distributed or hand drawing the icons. Photo documentation from the task is provided in Figure 4.

Overall, students seemed to find the task easy to approach and most groups finished in time. Students were asked to leave the prints after the workshop for us to use in the further development of the cards. From this, we could see that not all cards had been assigned phases, which we assess as an indication of the students not always fully understanding the content of the cards. For most material positions there 
was coherence in how groups had assigned phases, here we consider this as at least four out of the seven groups, with the 'Manufacturing and Production' and the 'Design and Concept' phases being the most represented and 'Transport and Retail' being the least and almost not used. For us, these inputs and insights were valuable as guidelines for us to assign the phases in the 'final' version of the deck.
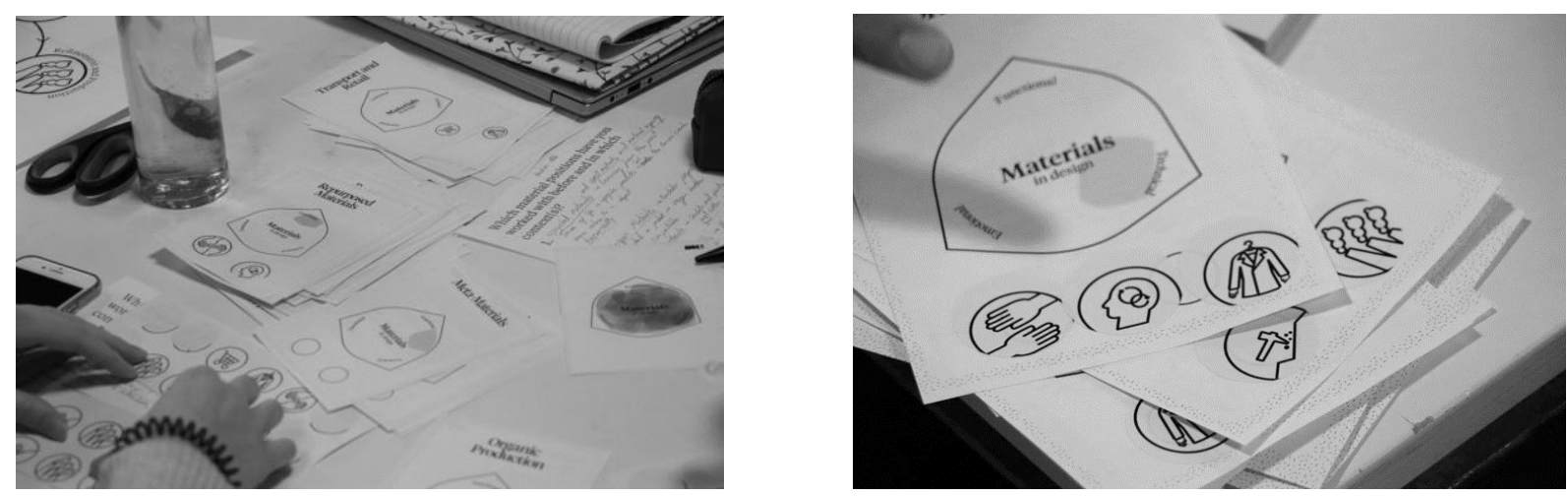

Figure 4. Linking material positions to phases in the product lifecycle

\subsection{Revising the cards}

The above-described concrete insights from the workshop as well as from oral feedback afterwards -the students were overall positive towards using the deck - motivated us to revise the cards. For example, by means of how the content on the cards was framed and described, if we should consider to merge or split some material positions and generally how we saw the deck being positioned in the world. Furthermore, it also meant clarifying that the cards are not and should not be seen as value-laden guidelines or methods, but as neutral material positions in the world that support that materials' engagement with sustainability can have positive as well as negative impact.

Based on this, the position titles were aligned, with exception of two cards, using 'Material' as the first word. Furthermore, the number of cards in the deck was cut down from 33 to 22, for example by merging cards that related to circularity into one position called 'Material circulation' and related to degradation into one position called 'Material degradation'.

\section{THE MATERIAL PATHWAYS DECK}

After the overall structure of the cards was settled, the content of each card was continuously re-written and revised until the cards were printed as a physical deck in the fall 2020. As shown in figure 5, the material positions represent all areas of the design compass (left) and all material positions have (strong) links to other positions (right).
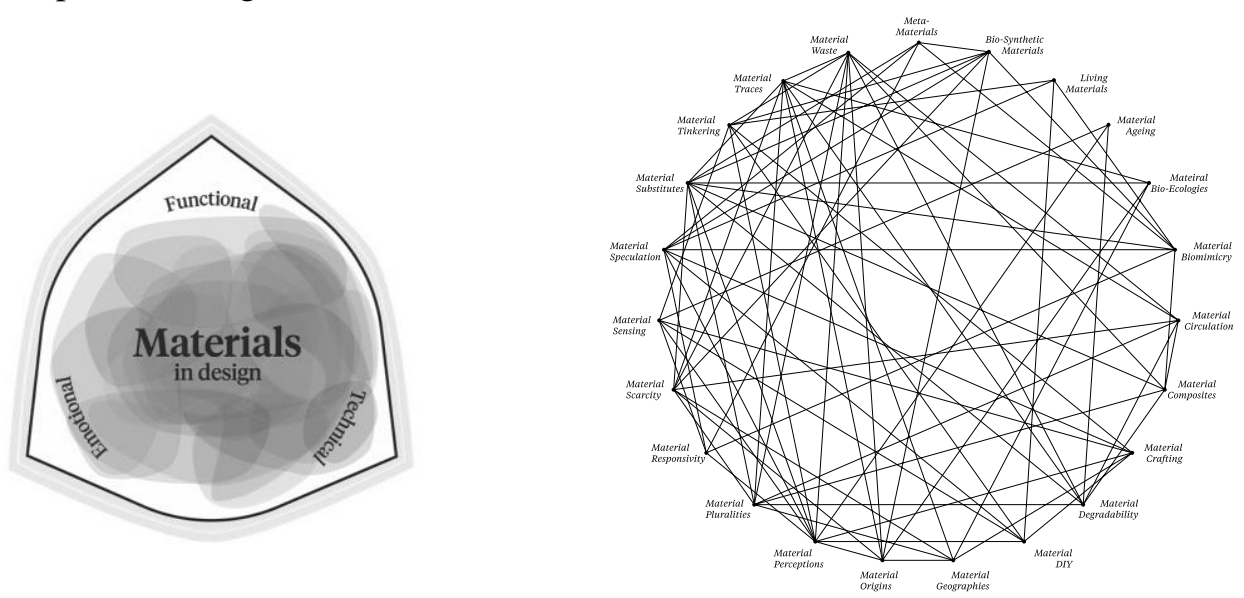

Figure 5. The design compass including the 22 material positions (left), an affinity diagram showing relations between the 22 material positions (right)

In figure 6, the material position 'Material Ageing' is included as a card example. The graphics side shows that the position is linked to the lifecycle phases 'User and Practice' and 'Design and Concept' 
and that it, according to the design compass, is positioned in the space between technical and emotional lifetime.
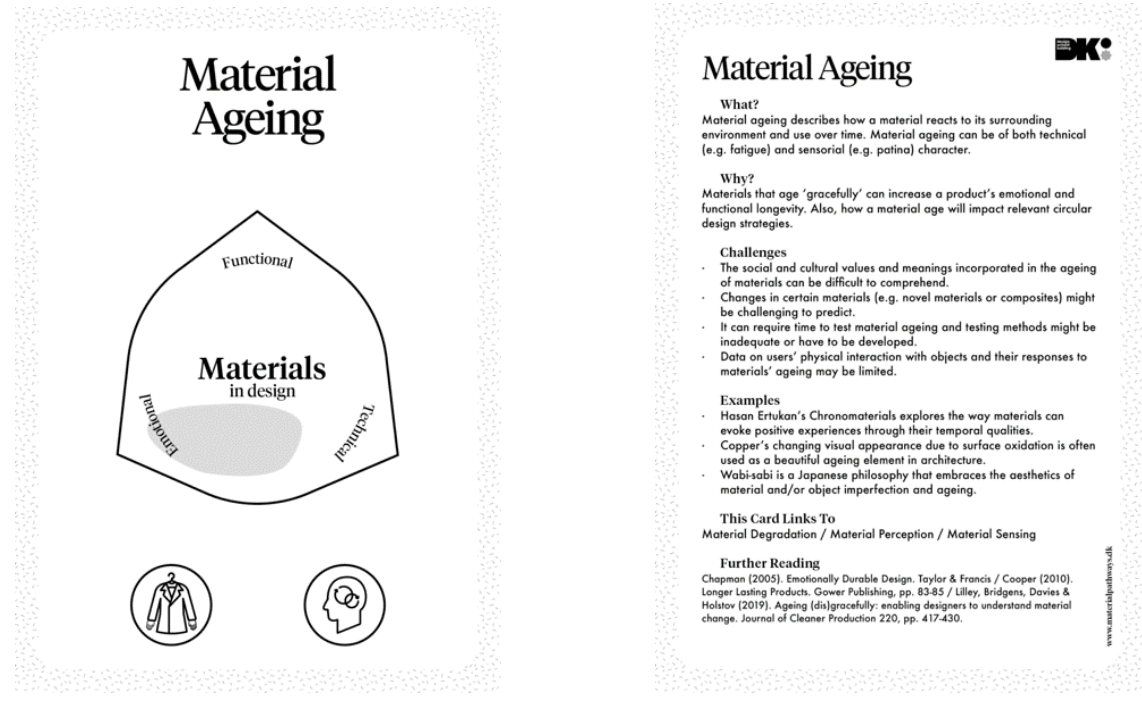

Figure 6. Example of the card, Material Ageing with a graphics side (left) and information side (right)

\section{WHAT COMES NEXT?}

We are now interested in understanding how the cards might be adopted within traditional design disciplines to further material engagement and reflection. Our own MA program, XX that comprises five disciplines. It thus provides us a rich context to explore this question. However, given the inter and cross disciplinary nature of the program, it also brings an opportunity to understand how the deck might be useful for students when they develop and define material concepts and approaches a cross traditional disciplinary material understandings and traditions.

Second, we believe (as was found with the Sustainable Design Cards), that the Material Pathways deck might hold relevance for disciplinary practices affiliated to design such as architecture, design management, material and product engineering and business studies. We speculate, that by broadening the scope of what materials in design can mean in relation to sustainability by including and explicating notions and concepts from human, social and natural sciences, the tool might potentially be able to bridge knowledge domains and bring nuances to discussions on the role of materials in the world.

\section{REFERENCES}

[1] Hasling K. M. and Ræbild U. 'Sustainability Cards: Design for Longevity', in Proceedings of PLATE 2017 - Product Lifetimes and the Environment, Delft, the Netherlands, 2017, pp. 166170.

[2] Ræbild U. and Hasling K. M. 'Experiences of the Sustainable Design Cards: evaluation of applications, potentials and limitations', Fash. Pract. J. Des. Creat. Process Fash. Ind., vol. 11, no. 3, pp. 417-442, 2019, doi: https://doi.org/10.1080/17569370.2019.1664026.

[3] Ræbild U. and Hasling K. M. 'Sustainable Design Cards: A Learning Tool for Supporting Sustainable Design Strategies', in Sustainable Fashion in a Circular Economy, Niinimäki K., Ed. Helsinki: Aalto University, 2018, pp. 128-151.

[4] Cooper T. 'Slower Consumption - Reflections on Product Life Spans and the "Throwaway Society", J. Ind. Ecol., vol. 9, no. 1-2, pp. 51-68, 2005.

[5] Ellen MacArthur Foundation, 'Towards the Circular Economy vol. 1', Ellen MacArthur Foundation, 1, 2012.

[6] Ashby M. F. 'End of first life: a problem or a resource (Chapter 4)', in Materials and the environment: eco-informed material choice, Butterworth-Heinemann, 2013, pp. 79-97. 